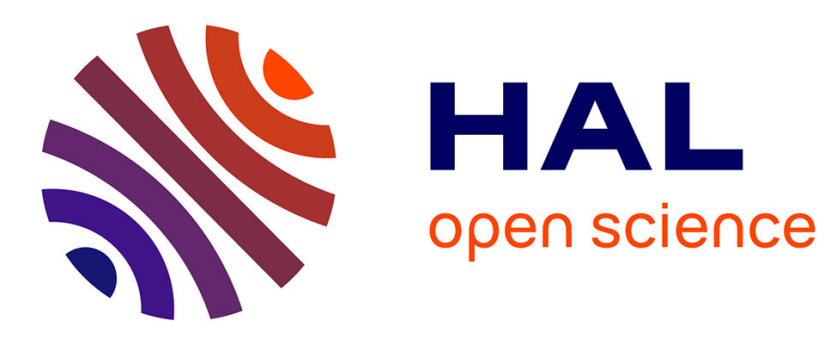

\title{
Sur la polarisation elliptique
}

\author{
L. Mouton
}

\section{To cite this version:}

L. Mouton. Sur la polarisation elliptique. J. Phys. Theor. Appl., 1875, 4 (1), pp.240-245. 10.1051/jphystap:018750040024001 . jpa-00237066

\section{HAL Id: jpa-00237066 https://hal.science/jpa-00237066}

Submitted on 1 Jan 1875

HAL is a multi-disciplinary open access archive for the deposit and dissemination of scientific research documents, whether they are published or not. The documents may come from teaching and research institutions in France or abroad, or from public or private research centers.
L'archive ouverte pluridisciplinaire HAL, est destinée au dépôt et à la diffusion de documents scientifiques de niveau recherche, publiés ou non, émanant des établissements d'enseignement et de recherche français ou étrangers, des laboratoires publics ou privés. 


\section{SUR LA POLARISATION ELLIPTIQUE;}

Par L. MOUTON,

Agrégé-Préparateur à l’École Normale.

On sait qu'un rayon elliptique peut être considéré comme résultant de la composition de deux rayons polarisés rectilignement dans la direction des axes de l'ellipse et présentant une différence de phase de $\frac{\pi}{2}$ ou, ce qui revient au même, une différence de marche de $\frac{\lambda}{4}, \lambda$ étant la longueur d'onde de la lumière considérée $\left({ }^{1}\right)$.

(') De Senarmont, Annales de Chimie et de Physique, 2 érie, t. LXIII, p. 345; JAmis, idem, 3e série, t. XIX, p. $32 \mathrm{I}$ et suiv.; JAxIx, Cours de Physique, t. III, p. 63ı; Billet, Traité d'Optique phrsique, t. II, p. 56. 
Il suit de là qu'une lame mince d'épaisseur telle, qu'elle introduise, entre le rayon polarisé dans sa section principale et le rayon polarisé perpendiculairement, une différence de marche de $\frac{\lambda}{4}$, placée normalement sur le trajet d'un rayon elliptique, le ramènera à la polarisation rectiligne, quand sa section principale coïncidera arec l'un des axes de l'ellipse.

C'est un procédé qu'a employé de Senarmont pour déterminer les éléments d'un rayon elliptique.

La difficulté de tailler une lame exactement quart d'onde pour une lumière donnée, l'obligation où l'on est ensuite de ne l'employer que pour cette lumière conduisent à se demander ce que produirait, sur un rayon elliptique de longueur d'onde donnée, l'interposition d'une lame mince un peu diflérente du quart d'onde de cette lumière.

Voyons pour cela comment varie la différence de phase du système rectangulaire constituant le rayon elliptique, lorsque ce système tourne autour du centre de l'ellipse.

Fig. 1.

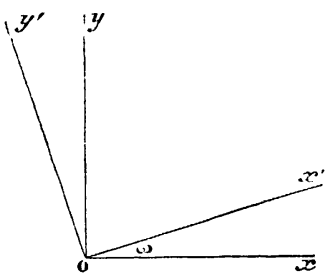

Pour plus de simplicité, partons avec M. Jamin du mouvement rapporté aux axes de l'ellipse ( ${ }^{1}$ ); on a

$$
\begin{aligned}
& x=a \cos 2 \pi \frac{t}{T}, \\
& y=b \cos \left(2 \pi \frac{t}{T}-\frac{\pi}{2}\right)=b \sin \pi \frac{t}{T} .
\end{aligned}
$$

Si l'on fait tourner les axes d'un angle $\omega$, qu'on applique les formules de transformation connues et qu'on mette les mourements

(') Cours de Physique, t. III, P. 632.

J. de Phys., t. IV. (Aoùt is $\$$ j.) 
sous la forme ordinaire,

$$
\begin{aligned}
& x^{\prime}=\mathbf{A} \cos \left(2 \pi \frac{t}{\mathbf{T}}+o^{\prime}\right), \\
& y^{\prime}=\mathbf{B} \cos \left(2 \pi \frac{t}{\mathbf{T}}+o^{\prime \prime}\right),
\end{aligned}
$$

on a

$$
\operatorname{tang}\left(\delta^{\prime}-\delta^{\prime \prime}\right)=-\frac{2 a b}{a^{2}-\overline{b^{2}}}-\frac{1}{\sin 2 \omega} \cdot
$$

La discussion de ce résultat n'offre aucune difficulté. Elle inontre que l'arc $\partial^{\prime}-\partial^{\prime \prime}$, partant de $\frac{\pi}{2}$ pour $\omega=0$, croit jusqu'à une certaine valeur $\mathrm{ABY}$, dont la tangente est $\frac{2 a b}{a^{2}-\frac{a b}{2}}$, et qui correspond

Fig. 2.

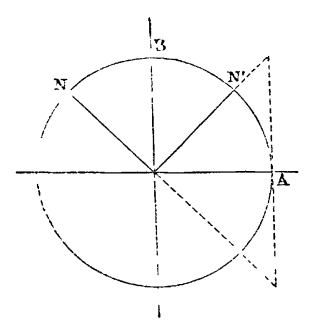

à $\omega==45^{\circ}$; il revient ensuite à $\frac{T}{2}$ pour $\omega=90^{\circ}$, décroît jusqu'à $A N^{\prime}$ de tangente $\frac{2 a b}{a^{2}-b^{2}}$ qu'il atteint pour $\omega=4 \tilde{J}^{\circ}+90^{\circ}$, et retourne enfin a $\frac{\pi}{2}$ pour $\omega=180^{\circ}$. De plus, l'arc $\delta^{\prime}-\sigma^{\prime \prime}$ reprend les mèmes valeurs pour $\omega=45^{\circ} \pm \alpha$, ou $\omega=135^{\circ} \pm x$.

En résumé, la différence de phase prend une valeur maximum et un minimum, tous deux également distants de $\frac{\pi}{2}$, l'un en plus, l'autre en moins; elle les atteint lorsque le système d'axes se confond avec les bissectrices des axes de l'ellipse, et elle prend les mêmes valeurs pour deux orientations également inclinées sur ces bissectrices $\left({ }^{1}\right)$.

(') Billet, Traité d'Optique physique, p.64́, donne un tableau où se constatent facilement ces résultats. 
Il résulte de ce qui précède que, si sur le trajet d'un rayon clliptique on interpose une lame mince plus voisine de $\frac{\pi}{2}$ queles valeurs extrèmes que peut prendrel'anomalie des composantes de ce rayon, on trouvera deux orientations de cette lame pour lesquelles la polarisation plane sera rétablie, et elles seront également inclinées sur l'une des bissectrices des axes de l'ellipse.

Sans que j'insiste davantage, on voit qu'une plaque mince trèspeu supérieure au $\frac{\lambda}{4}$ rouge, par exemple, pourra servir dans la grande majorité des cas; la lumière elliptique à laquelle elle ne s’appliquerait pas serait circulaire ou à peu près.

Reste à voir comment nous tirerons de là les éléments que l'on détermine ordinairement du rayon elliptique.

Les limites imposées à cet article m’obligent à ètre bref: je ne donnerai que les calculs, sans insister sur des détails que l'on complétera sans peine.

Fig. 3.

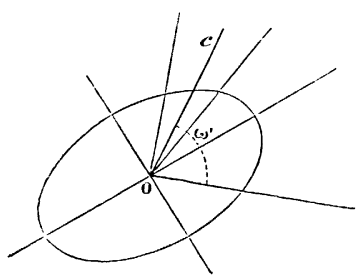

Soient $\omega_{1}$ el $\omega_{2}$ les angles que font, avec une direction quelconque OX prise pour origine, deux orientations autour de la bissectrice $\mathrm{OC}$, pour lesquelles notre plaque a rétabli la polarisation rectiligne; l'angle $\omega^{\prime}$ de OC avec OX sera $\omega^{\prime}=\frac{\omega_{1}+\omega_{2}}{2}$.

Or, si nous appelons $\delta$ la diflérence de phase sclon deux plans principaux donnés, suivant lesquels nous décomposons les mourcments elliptiques, et tang $\%$ le rapport $\frac{b}{a}$ des amplitudes suivant ces deux plans, on a la relation

$$
\cot 2 \omega^{\prime}=-\operatorname{tang} 2 \alpha \cos o^{\prime}(),
$$

(') J⿳一巛工 
d'où, dans le cas actuel,

$$
\cot \left(\omega_{1}+\omega_{2}\right)=-\operatorname{tang} 2 \alpha \cos \delta .
$$

Mais le Nicol analyseur, en même temps qu'il nous indiquera le rétablissement de la polarisation rectiligne dans l'azimut $\omega_{1}$, par exemple, nous donnera l'angle $\bigcup_{1}$ que fait la vibration rectiligne rétablie avec la direction $\omega_{1}$. Or, si l'on appelle $A_{1}$ et $B_{1}$ les amplitudes rapportées à cet azimut $\omega_{1}$, on a la relation connue

avec les suivantes $\left({ }^{1}\right)$ :

$$
\operatorname{tang}^{2} \Psi_{1}=\frac{\mathbf{B}_{1}^{2}}{\mathbf{A}_{1}^{2}},
$$

$$
\left\{\begin{array}{l}
\mathbf{A}_{1}^{2}=\sin ^{2} \alpha \sin ^{2} \omega_{1}+\cos ^{2} \alpha \cos ^{2} \omega_{1}+\frac{1}{2} \sin 2 \alpha \sin 2 \omega_{1} \cos \delta, \\
\mathbf{B}_{1}^{2}=\sin ^{2} \alpha \cos ^{2} \omega_{1}+\cos ^{2} \alpha \sin ^{2} \omega_{1}-\frac{1}{2} \sin 2 \alpha \sin 2 \omega_{1} \cos \delta,
\end{array}\right.
$$

et nous sommes finalement conduits à tirer du système formé par les trois équations ci-dessus et celle trouvée plus haut,

$$
\cot \left(\omega_{1}+\omega_{2}\right)=-\operatorname{tang} 2 \alpha \cos \delta,
$$

les valeurs de $\alpha$ et de $\delta$ en fonction des données d'observation $\omega_{1}$, $\omega_{2}$ et $\psi_{1}$.

Avant de faire cette détermination, je ferai remarquer qu'à l'orientation $\omega_{2}$ correspond aussi un angle $\psi_{2}$, analogue à $\psi_{1}$; mais on voit sans peine que cet angle $\psi_{2}$ est complémentaire de $\psi_{1}$, et qu'il nous conduirait à des relations rentrant dans les précédentes.

Reste donc à résoudre le système précédent.

La première des équations (2) peut s'écrire

$$
\frac{1-\operatorname{tang}^{2} \Psi_{1}}{I+\operatorname{tang}^{2} \Psi_{1}}=\frac{A_{1}^{2}-B_{1}^{2}}{A_{1}^{2}+B_{1}^{2}}=\frac{A_{1}^{2}-B_{1}^{2}}{I}
$$

ou

$$
\cos 2 \psi_{1}=\mathbf{A}_{1}^{2}-\mathbf{B}_{1}^{2},
$$

et, d'après les deux autres,

$$
\cos 2 \psi_{1}=\cos 2 \omega_{1} \cos 2 \alpha+\sin 2 \alpha \sin 2 \omega_{1} \cos \delta,
$$

relation à laquelle il faut joindre

$$
\cot \left(\omega_{1}+\omega_{2}\right)=-\operatorname{tang} 2 \alpha \cos \delta .
$$

(') Jamis, Annales de Chimie et de Physique, 3e série, t. XIX, p. 323. 
De la première, en tenant compte de celle-ci, on tire

$$
\begin{aligned}
\frac{\cos 2 \psi_{1}}{\cos 2 \alpha} & =\cos 2 \omega_{1}-\sin 2 \omega_{1} \cot \left(\omega_{1}+\omega_{2}\right) \\
& =\cos 2 \omega_{1}-\sin 2 \omega_{1} \frac{\cos 2 \omega_{1}+\cos 2 \omega_{2}}{\sin 2 \omega_{1}+\sin 2 \omega_{2}} \\
& =\frac{\sin 2\left(\omega_{2}-\omega_{1}\right)}{\sin 2 \omega_{1}+\sin 2 \omega_{2}}=\frac{2 \sin \left(\omega_{2}-\omega_{1}\right) \cos \left(\omega_{2}-\omega_{1}\right)}{2 \sin \left(\omega_{2}+\omega_{1}\right) \cos \left(\omega_{2}-\omega_{1}\right)} \\
& =\frac{\sin \left(\omega_{2}-\omega_{1}\right)}{\sin \left(\omega_{2}+\omega_{1}\right)}
\end{aligned}
$$

d'où

$$
\cos 2 \alpha=\frac{\cos 2 \psi_{1} \sin \left(\omega_{2}+\omega_{1}\right)}{\sin \left(\omega_{2}-\omega_{1}\right)}
$$

formule calculable par logarithmes et qui donnera $\alpha$; on tirera ensuite $\delta$.

Je n'insiste pas sur les questions de détail, telles que signes, etc.; je renvoie pour cela au Mémoire de de Senarmont, où ces difficultés secondaires se présentent et se résolvent d'une manière analogue.

On devine sans peine comment ce procédé, qui revient en fin de compte à éliminer le retard dû à la lame mince, s'applique même à une lumière non homogène. Que l'analyseur soit en effet suivi d'un spectroscope, quand une bande noire apparaitra dans le spectre à la place d'une couleur déterminée, les calculs précédents nous donneront la différence de phase et le rapport des intensités des composantes du rayon elliptique de cette couleur.

L'idée de cette extension de la méthode de de Senarmont appartient à M. Eilhard Wiedemann, qui l'a appliquée à des études de lumière réfléchie; on trouvera dans son Mémoire $\left({ }^{1}\right)$ tous les détails de son expérimentation, des résultats de laguelle il a été rendu compte dans le Journal de Ply sique. Seulement II. Wiedemann n'arrive aux formules qui lui permettent de calculer la différence de phase et le rapport des amplitudes des deux composantes du rayon elliptique que par un calcul incontestablement original, mais long et de toutes pièces; $j$ 'ai pensé qu'il ne serait peut-ètre pas sans intérêt de montrer qu'on arrive à des résultats mème plus simples par une application toute naturelle des formules des physiciens français.

(') Annales de Poggendorff, t. CLIII. 